University of Wollongong

Research Online

Faculty of Informatics - Papers (Archive)

Faculty of Engineering and Information

Sciences

$1-1-2010$

\title{
Multi-resolution mean-shift algorithm for vector quantization
}

P L. M Bouttefroy

philippe@uow.edu.au

A Bouzerdoum

University of Wollongong, bouzer@uow.edu.au

A Beghdadi

S L. Phung

University of Wollongong, phung@uow.edu.au

Follow this and additional works at: https://ro.uow.edu.au/infopapers

Part of the Physical Sciences and Mathematics Commons

\section{Recommended Citation}

Bouttefroy, P L. M; Bouzerdoum, A; Beghdadi, A; and Phung, S L.: Multi-resolution mean-shift algorithm for vector quantization 2010, 523-523.

https://ro.uow.edu.au/infopapers/832

Research Online is the open access institutional repository for the University of Wollongong. For further information contact the UOW Library: research-pubs@uow.edu.au 


\title{
Multi-resolution mean-shift algorithm for vector quantization
}

\begin{abstract}
The generation of stratified codebooks, providing a subset of vectors at different scale levels, has become necessary with the emergence of embedded coder/decoder for scalable image and video formats. We propose an approach based on mean-shift, invoking the multi-resolution framework to generate codebook vectors. Applied to the entire image, mean-shift is slow because it requires each sample to converge to a mode of the distribution. The procedure can be sped up with three simple assumptions: kernel truncation, code attraction and trajectory attraction. Here we propose to apply the mean-shift algorithm to the four image subbands generated by a DWT, namely the LL, LH, HL and HH subbands. It can be concluded from experimental results that the proposed MR-MS achieves similar PSNR to the LBG algorithm but outperforms it in terms of computation time.
\end{abstract}

\section{Keywords}

resolution, quantization, mean, multi, shift, vector, algorithm

Disciplines

Physical Sciences and Mathematics

\section{Publication Details}

Bouttefroy, P. L. M., Bouzerdoum, A., Beghdadi, A. \& Phung, S. L. (2010). Multi-resolution mean-shift algorithm for vector quantization. DCC 2010: 2010 Data Compression Conference (pp. 523-523). USA: IEEE. 


\title{
Multi-resolution Mean-shift Algorithm for Vector Quantization
}

\author{
P.L.M. Bouttefroy ${ }^{1,2}$, A. Bouzerdoum ${ }^{1}$, A. Beghdadi ${ }^{2}$, S.L. Phung ${ }^{1}$ \\ ${ }^{1}$ SECTE, University of Wollongong, Northfields Av., NSW 2522, Australia \\ ${ }^{2}$ L2TI, Université Paris 13, 99 Av. J.-B. Clément, 93430 Villetaneuse, France \\ \{philippe, a.bouzerdoum,phung\}@uow.edu.au, beghdadi@univ-paris13.fr
}

The generation of stratified codebooks, providing a subset of vectors at different scale levels, has become necessary with the emergence of embedded coder/decoder for scalable image and video formats. We propose an approach based on mean-shift, invoking the multi-resolution framework to generate codebook vectors. Applied to the entire image, mean-shift is slow because it requires each sample to converge to a mode of the distribution. The procedure can be sped up with three simple assumptions: kernel truncation, mode attraction and trajectory attraction.

Here we propose to apply the mean-shift algorithm to the four image subbands generated by a DWT, namely the LL, LH, HL and HH subbands. The simulated annealing technique traditionally used to identify the modes of the distribution for different resolutions can be performed by exploration of the multi-scale DWT pyramid, avoiding the costly estimation by a full mean-shift at each level. The proposed technique, called multi-resolution mean-shift (MR-MS) is described below.

1) At the $i$ th level of decomposition, the modes of the LL $i$ subband are obtained by mean-shift to generate a coarse codebook. This procedure is fast since it is performed on an image with reduced number of pixels.

2) A threshold on the high-pass subbands $\mathrm{LH} i, \mathrm{HL} i$ and $\mathrm{HH} i$ is applied to detect salient pixels. Located in regions with high frequencies, these pixels are likely to uncover new modes in the distribution at a lower level of decomposition.

3) A modified mean-shift is run on the set of salient pixels and new vectors are added to the codebook. The modified mean-shift includes the modes of the distribution from higher levels of decomposition with a weight proportional to the number of pixels in the basin of attraction so that the existing vectors are represented according to their importance. The procedure is very fast because it is limited to high-frequency regions and existing modes.

4) This generates the codebook for subband $\mathrm{LL}_{i-1}$. The process can be iterated, starting from Step 2 above to generate a new set of codebook vectors for the next level.

Experiments were run on a dataset comprising some benchmark images (e.g. Lena, Baboon, Peppers, etc.) and some high resolution images. The aim is to compare the MR-MS with the LBG algorithm. The mean-shift performance is also displayed. However, it does not allow stratified codebooks. Codebooks of size 512, 1024 and 2048 were generated. Table I presents the results in terms of PSNR and processing time. It can be concluded that the MR-MS achieves similar PSNR to the LBG algorithm but outperforms it in terms of computation time.

Table I

PSNR AND COMPUTATION TIME FOR MR-MS AND LBG

\begin{tabular}{|c|c|c|c|c|c|c|}
\cline { 2 - 7 } \multicolumn{1}{c|}{} & \multicolumn{3}{c|}{ PSNR (in dB) } & \multicolumn{3}{c|}{ Time (in sec.) } \\
\cline { 2 - 7 } \multicolumn{1}{c|}{} & Mean-shift & MR-MS & LBG & Mean-shift & MR-MS & LBG \\
\hline 512 & 30.1 & 29.9 & 30.6 & 11.2 & 9.36 & 111 \\
\hline 1024 & 31.7 & 32.0 & 31.5 & 18.5 & 16.4 & 214 \\
\hline 2048 & 33.6 & 33.9 & 34.4 & 40.5 & 33.5 & 421 \\
\hline
\end{tabular}

